\title{
Ocular delivery of natamycin based on monoolein/span 80/poloxamer 407 nanocarriers for the effectual treatment of fungal keratitis
}

\author{
Marzuka KAZI *1 (D), Renuka DHAKNE 2 (D), Mohamed Hassan DEHGHAN ${ }^{3}$ (D) \\ 1 Department of Pharmaceutics, Faculty of Y.B. Chavan College of Pharmacy, Dr. Rafiq Zakaria Campus, \\ Aurangabad (M.S.), India. \\ 2 Department of Pharmaceutics, Faculty of Yash Institute of Pharmacy, Aurangabad (M.S.), India \\ 3 Department of Pharmaceutics, Faculty of Y.B. Chavan College of Pharmacy, Dr. Rafiq Zakaria Campus, \\ Aurangabad (M.S.), India. \\ * Corresponding Author. E-mail: marzi345@gmail.com (M.K.); Tel. +91-7045-349 259.
}

Received: 01 October 2019 / Revised: 22 January 2020 / Accepted: 03 February 2020

\begin{abstract}
A 32 factorial design was used to develop Natamycin cubosome nanoparticles with enhanced corneal permeation, so as to effectively treat ocular fungal keratitis. Probe sonication technique was deployed to disperse the dry lipidic film to obtain colloidal dispersion. The colloidal dispersion was characterized for critical quality attributes such as particle size, poly dispersibility index (PDI), zeta potential and entrapment efficiency. The optimized batch exhibited a particle size of $158.2 \mathrm{~nm}$, zeta potential $-40 \mathrm{mV}$, PDI 0.328 in addition, entrapment efficiency of $99.85 \%$. The in vitro drug release of natamycin from optimized cubosome demonstrated a cumulative \%drug release of $84.29 \%$ at the end of 8 hours. The optimized cubosomal dispersion exhibited enhanced in vitro antifungal activity against Candida albicans and Aspergillus fumigatus as compared to a pure drug suspension. The optimized formulation was further analyzed for polarized light microscopy (PLM), transmission electron microscopy (TEM) and small angle Xray scattering (SAXS) to state the morphology of formed cubosome nanoparticles and was noted to be $\operatorname{Im} 3 \mathrm{~m}$ bicontinous cubic mesophasic structure. X-ray diffraction (XRD) studies affirmed the complete encapsulation of natamycin into cubosome vesicles. Ex vivo corneal permeation studies of optimized formulation revealed enhanced corneal permeation in comparison to a pure drug suspension. The ocular irritation studies performed on rabbits indicated the cubosome to be non-irritant. Finally, the developed natamycin cubosome nanoparticles demonstrated sustained drug release and increased corneal penetration. Thus, these cubosome nanocarriers present a propitious delivery system for effective management of ocular fungal keratitis.
\end{abstract}

KEYWORDS: Natamycin; cubosome; ocular fungal keratitis; nanoparticles; factorial design.

\section{INTRODUCTION}

Fungal keratitis is a severe ocular infection characterized by decreased vision, photophobia, featheryedged infiltrates and satellite lesions across the cornea that leads to cataclysmic visual fallouts [1]. The causative agent is a species of fungi such as yeasts (Candida), filamentous with septae (Aspergillus, Fusarium, Cladosporium, Curvularia) and filamentous with non-septated (Rhizopus) [2]. Natamycin is BCS class II drug and due to its high molecular weight, corneal permeation is low. The conventional therapy for natamycin surfaces some notable drawbacks such as high dosing frequency, longer time period treatment cycles (4-6 weeks) and due to fast removal by nasopharyngeal drainage residence time at the ocular mucosa is short [3]. Extensive efforts have been directed towards the enhancement of ophthalmic drug bioavailability by exploring novel drug delivery strategies [3]. The delivery alternatives aim at improving both the precorneal residence time and in boosting the trans-corneal permeation of the drug $[4,5]$. Thus, the challenge faced in designing a successful delivery can only be overcomed by circumventing the shielding barriers of the eye without enduring any tissue injury.

Nanocarriers or nanoparticulate systems proposed for controlled drug delivery demonstrate ability as a potential strategy to overcome these limitations [6]. Recently, cubosomes as a surrogate nanocarrier drug delivery system to liposome has been investigated [7]. These are binary systems, especially made up monoolein-water and are three-dimensional nanostructure with hydrophobic and hydrophilic domains that

How to cite this article: Kazi M, Dhakne R, Dehghan $\mathrm{MH}$. Development of natamycin nanocarriers for effectual treatment in ocular fungal keratitis. J Res Pharm. 2020; 24(2): 251-263. 
can self-order into thermodynamically stable bicontinuous cubic liquid crystalline phases [8]. These are capable of loading and enhancing the penetration of lipophilic, hydrophilic, and amphiphilic drugs [9].

The present investigation focuses on the development of Natamycin cubosome nanoparticles by implementing a $3^{2}$ factorial design. The study seeks to enhance corneal permeation of the drug, to develop a drug delivery system that could aid in effectively treating ocular fungal keratitis.

\section{RESULTS AND DISCUSSION}

\subsection{Formulation and optimization of cubosomes using $3^{2}$ factorial design}

A $3^{2}$ factorial design was constructed to statistically study the prime effects of each factor and their interaction on the characteristics of cubosome nanoparticles. The independent variables selected were the concentration of span $80\left(\mathrm{X}_{1}\right)$, it was used as the lipidic surfactant in conjunction with glycerol monooleate (GMO) and concentration of poloxamer 407 (P-407) $\left(\mathrm{X}_{2}\right)$ which functioned as a stabilizer to form unaggregated small size particles. The effect of independent variables was evaluated to extrapolate its effect on critical quality attributes (CQAs) such as mean particle size $\left(\mathrm{Y}_{1}\right)$, zeta potential $\left(\mathrm{Y}_{2}\right)$, polydispersibility index (PDI) $\left(\mathrm{Y}_{3}\right)$ and entrapment efficiency $\left(\mathrm{Y}_{4}\right)$ and is presented in Table 1. The statistical analysis of critical quality responses is presented in Table 2 .

Table 1. Effect of independent factors on dependent responses (CQAs).

\begin{tabular}{|c|c|c|c|c|c|c|}
\hline \multirow[b]{2}{*}{ Formulations } & \multicolumn{2}{|c|}{$\begin{array}{c}\text { Independent } \\
\text { factors }\end{array}$} & \multicolumn{4}{|c|}{ Dependent responses } \\
\hline & $\begin{array}{c}X_{1} \\
(\mathrm{mg})\end{array}$ & $\begin{array}{c}X_{2} \\
(\mathrm{mg})\end{array}$ & $\mathrm{Y}_{1}$-Mean particle size $(\mathrm{nm})$ & $\begin{array}{c}\mathrm{Y}_{2} \text {-Zeta } \\
\text { potential } \\
(\mathrm{mV})\end{array}$ & $\begin{array}{l}\mathbf{Y}_{3^{-}} \\
\text {PDI }\end{array}$ & $\begin{array}{c}\mathrm{Y}_{4^{-}} \\
\text {Entrapment } \\
\text { efficiency } \\
(\%)\end{array}$ \\
\hline F1 & 200 & 200 & 185.7 & -33.2 & 0.312 & 99.09 \\
\hline F2 & 200 & 50 & 209.2 & -28.6 & 0.528 & 99.64 \\
\hline F3 & 300 & 125 & 318.5 & -27.3 & 0.525 & 99.45 \\
\hline F4 & 100 & 200 & 174.1 & -36.2 & 0.217 & 98.44 \\
\hline F5 & 200 & 125 & 188.6 & -30.9 & 0.568 & 98.98 \\
\hline F6 & 100 & 125 & 171.8 & -36.8 & 0.497 & 98.4 \\
\hline F7 & 100 & 50 & 158.7 & -40 & 0.328 & 99.85 \\
\hline F8 & 300 & 200 & 188.6 & -32 & 0.22 & 99.62 \\
\hline F9 & 300 & 50 & 505.5 & -17.6 & 0.622 & 99.64 \\
\hline
\end{tabular}

The determination coefficient $\left(R^{2}\right)$ for the observed and the predicted values is essential for reviewing the statistical significance of the model. $\mathrm{R}^{2}$ for mean particle size, zeta potential, PDI and encapsulation efficiency and were $0.8731,0.9809,0.9714$, and 0.9490 respectively. Figure 1 represents the contour plots for the various dependent responses.

Particle size was sorted as a CQA since small particles can resist the loss of drug due to impulsive blinking of the eye. Moreover it provides higher ocular bioavailability by increasing permeation across ocular tissues and is less irritating to the ocular tissues [10]. The particle size for all the nine batches ranged between 158.7-505.5 nm, the least particle size was noted for F7 whereas highest size is been seen for F9. The particle size was significantly affected by the concentration of span 80, P-407 concentration and their interaction. Increase in span 80 concentration lead to increase in particle size was an increase in P-407 concentration lead to decline in particle size. Increase in span 80 concentration leads to an enhance hydrophobic interaction with GMO thus causes enlargement of particle size [11]. The role of stabilizers is of prime importance as it controls the particle size and stabilizes the dispersions of nanoparticle formulations [12]. İt has been reported elsewhere, that high concentration of P-407 leads to decrease in surface tension and stabilization of the system with the formation of smaller particles $[13,14]$. A surface 2FI model was applied to statistically evaluate the dependent response $Y_{1} . X_{1}$ had a positive effect whilst $X_{2}$ and $X_{1} X_{2}$ had a negative effect and the same is relevant from the deduced polynomial equation:

$$
Y_{1}=233.4+84.67 X_{1}-54.17 X_{2}-83.08 X_{1} X_{2}
$$


Table 2. Statistical evaluation of various dependent responses.

\begin{tabular}{|c|c|c|c|c|c|c|c|c|}
\hline \multirow{2}{*}{ Factors } & \multicolumn{2}{|c|}{$\begin{array}{c}\text { Y1-Mean particle } \\
\text { size }(\mathrm{nm})\end{array}$} & \multicolumn{2}{|c|}{$\begin{array}{c}\mathrm{Y}_{2} \text {-Zeta potential } \\
(\mathrm{mV})\end{array}$} & \multicolumn{2}{|c|}{$\mathrm{Y}_{3}$-PDI } & \multicolumn{2}{|c|}{$\begin{array}{l}Y_{4} \text {-Entrapment } \\
\text { efficiency (\%) }\end{array}$} \\
\hline & Coefficient & $\begin{array}{c}\mathrm{p}- \\
\text { value }\end{array}$ & Coefficient & $\begin{array}{c}\mathrm{p}- \\
\text { value }\end{array}$ & Coefficient & $\begin{array}{c}\mathrm{p}- \\
\text { value }\end{array}$ & Coefficient & $\begin{array}{c}\mathrm{p}- \\
\text { value }\end{array}$ \\
\hline$\beta_{0}$ & 233.41 & 0.0111 & -31.40 & 0.0001 & 0.58 & 0.0160 & 99.23 & 0.0405 \\
\hline$X_{1}$ & 84.67 & 0.0094 & 6.02 & $\begin{array}{c}< \\
0.0001\end{array}$ & 0.054 & 0.0534 & 0.34 & 0.0477 \\
\hline$X_{2}$ & -54.17 & 0.0471 & -2.53 & 0.0029 & -0.12 & 0.0061 & -0.33 & 0.0508 \\
\hline$X_{1} X_{2}$ & -83.08 & 0.0219 & -4.55 & 0.0005 & -0.073 & 0.0426 & 0.35 & 0.0792 \\
\hline $\mathrm{X}_{1}{ }^{2}$ & - & - & - & - & -0.068 & 0.1110 & - & - \\
\hline$X_{2^{2}}$ & - & - & - & - & -0.16 & 0.0135 & - & - \\
\hline Model & $2 \mathrm{FI}$ & & $2 \mathrm{FI}$ & & Quadratic & & $2 \mathrm{FI}$ & \\
\hline $\mathbf{R}^{2}$ & 0.8731 & & 0.9809 & & 0.9714 & & 0.9490 & \\
\hline $\begin{array}{l}\text { Adjusted } \\
\mathbf{R}^{2}\end{array}$ & 0.7969 & & 0.9695 & & 0.9538 & & 0.9389 & \\
\hline $\begin{array}{l}\text { Predicted } \\
\mathbf{R}^{2}\end{array}$ & 0.7542 & & 0.9214 & & 0.9244 & & 0.9238 & \\
\hline $\begin{array}{l}\text { Adequate } \\
\text { precision }\end{array}$ & 16.45 & & 27.63 & & 10.19 & & 14.58 & \\
\hline
\end{tabular}

$\beta_{0}$-constant

$\mathrm{X}_{1}$-concentration of span 80

$\mathrm{X}_{2}$-concentration of Polaxamer 407
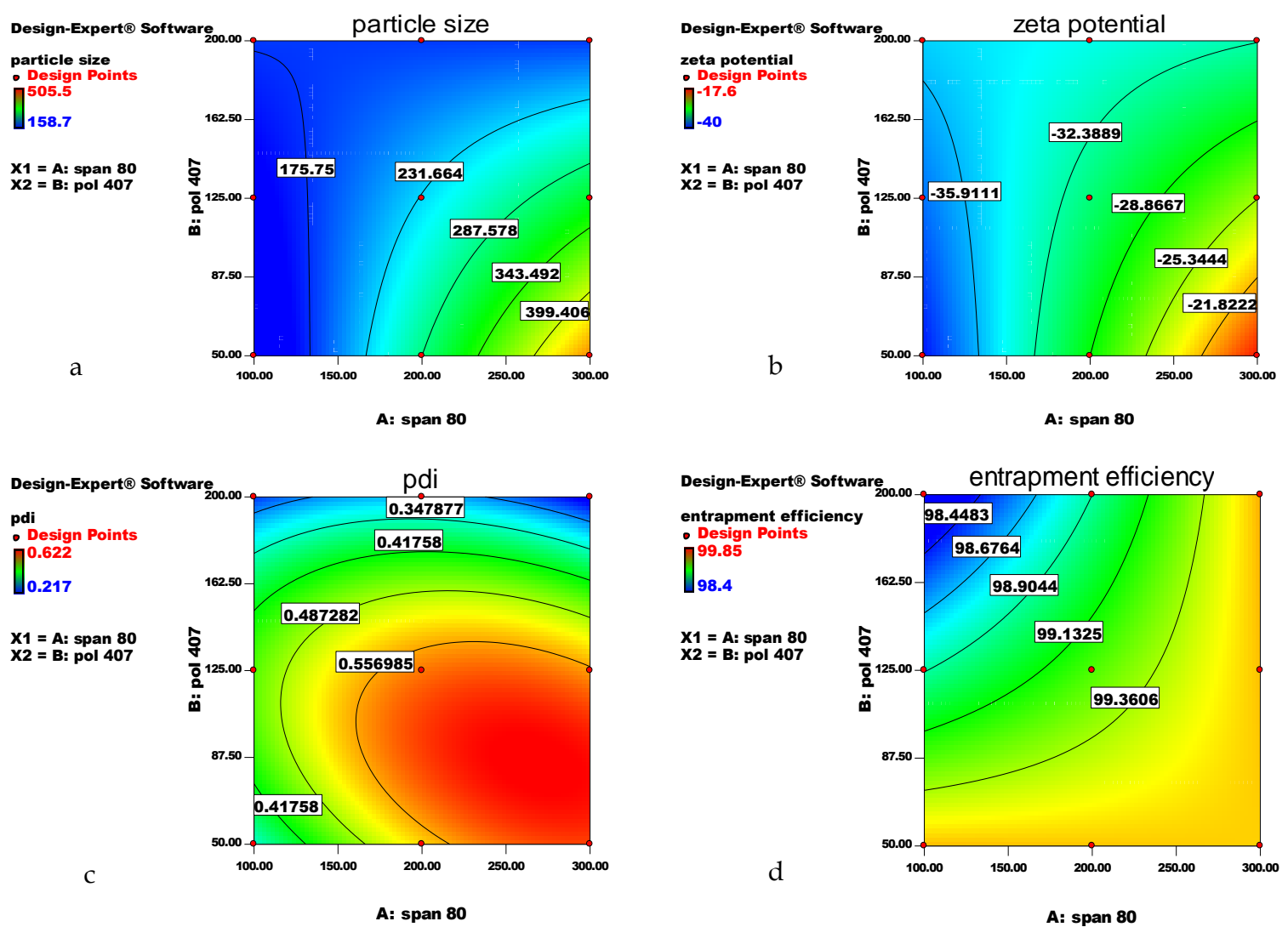

Figure 1. Contour plots presenting (a) the effect of concentration of polaxamer 407 and concentration of span 80 on mean particle size (b) the effect of concentration of polaxamer 407 and concentration of span 80 on zeta potential (c) the effect of concentration of polaxamer 407 and concentration of span 80 on PDI (d) the effect of concentration of polaxamer 407 and concentration of span 80 on entrapment efficiency. 
Zeta potential is also considered as a CQA in view of the fact that stability of colloidal dispersion is essential as the formulation would be presented as a suspension for ophthalmic use and thus also valuable for effective storage of the product during its shelf life. The zeta potential for the formulation was seen to be in the range -17.6 to $-40 \mathrm{mV}$. F7 exhibited the highest potential $(-40 \mathrm{mV})$ whereas the lowest potential $(-17.6$ $\mathrm{mV}$ ) was shown by F9. The negative value of $Y_{1}$ specifies the surface of the cubosome nanoparticles was negatively charged. A high zeta the potential value of cubosome nanoparticles highlights the extent of electrostatic repulsion among like-charged particles and is an indication of un-aggregated colloidal dispersion. $Y_{2}$ was significantly affected by $X_{1}, X_{2}$ and their interaction $X_{1} X_{2}$. İncrease in the concentration of $\mathrm{P}-407$ and interaction of $\mathrm{X}_{1} \mathrm{X}_{2}$ increased the average magnitude of zeta potential in contrast increase in span 80 concentration lead to a decrease, thus P-407 had a profound effect on stabilization of cubosomes [13]. A 2FI analysis model was used to statistical analysis the dependent variable $\left(\mathrm{Y}_{2}\right)$. The effect of independent variables on dependent response is mathematically modelled and represented by the following polynomial equation:

$$
Y_{2}=-31.40+6.02 X_{1}-2.53 X_{2}-4.55 X_{1} X_{2}
$$

PDI index gives a brief idea about particle size distribution and thus is also an important CQA. PDI values for the formulated batches was in the range 0.217-0.622. $X_{2}(p<0.05,0.0061), X_{1} X_{2}(p<0.05,0.0426)$ and $\mathrm{X}_{2}{ }_{2}(\mathrm{p}<0.05,0.0135)$ significantly affected PDI, and reduced the average PDI value. Whereas the other factors demonstrated a $\mathrm{p}$ value greater than 0.05 , thus had an insignificant effect on PDI. An quadratic model was applied to statistically evaluate the dependent variable $Y_{3}$. The response is mathematically expressed by the following quadratic equation:

$$
Y_{3}=0.58+0.054 X_{1}-0.12 X_{2}-0.073 X_{1} X_{2}-0.068 X_{1}^{2}-0.16 X_{2}^{2}
$$

Entrapment efficiency indicates the amount of drug encapsulated in the cubosome nanoparticle thus holds the key to the effective management of ocular fungal keratitis. $Y_{4}$ was impacted significantly by the concentration of span 80 , increase in $X_{1}(p<0.05,0.047)$ lead to higher \% entrapment, thus showcased positive effect. This may be due to more hydrophobic groups available for solubilizing of the drug with the expansion of the core radius [15]. The factors $X_{2}, X_{1} X_{2} \quad(p>0.05)$ had no significant effect on entrapment efficiency. Maximum entrapment was seen for F7 (99.85\%) as compared to F9 (98.4\%). A 2FI model was implemented to statistically examine the dependent variable $Y_{4}$. The response is expressed by the following polynomial equation:

$$
Y_{4}=99.23+0.34 X_{1}-0.33 X_{2}+0.35 X_{1} X_{2}
$$

The optimum formula of natamycin cubosome nanoparticles was calculated based on desirability function ( $\mathrm{d}$ value) upon target response. $\mathrm{D}$ value close to " 1 " signifies a desirable set of results and " 0 " is unfavourable. Average particle size (150-180 nm), zeta potential $(-20$ to $-40 \mathrm{mV})$, polydispersity index in range (0.28-0.385), and maximum entrapment efficiency $(>99 \%)$ were set as a limit. The design space was fixed at the lowest point for both concentrations of span 80 and P-407. Thus, with these set of pattern, a desirability value of 0.969 was achieved. The observed and predicted value for optimized cubosomes is presented in Table 3. The overlaid contour plots were drawn and are depicted in Figure 2.

Table 3. Predicted and observed dependent responses for optimized formulation.

\begin{tabular}{lll}
\hline Response & Predicted & Observed \\
\hline Mean particle size (nm) & 149.95 & 158.7 \\
Zeta potential (mV) & -37.53 & -40 \\
PDI & 0.388 & 0.328 \\
Entrapment efficiency (\%) & 99.57 & 99.85 \\
\hline
\end{tabular}




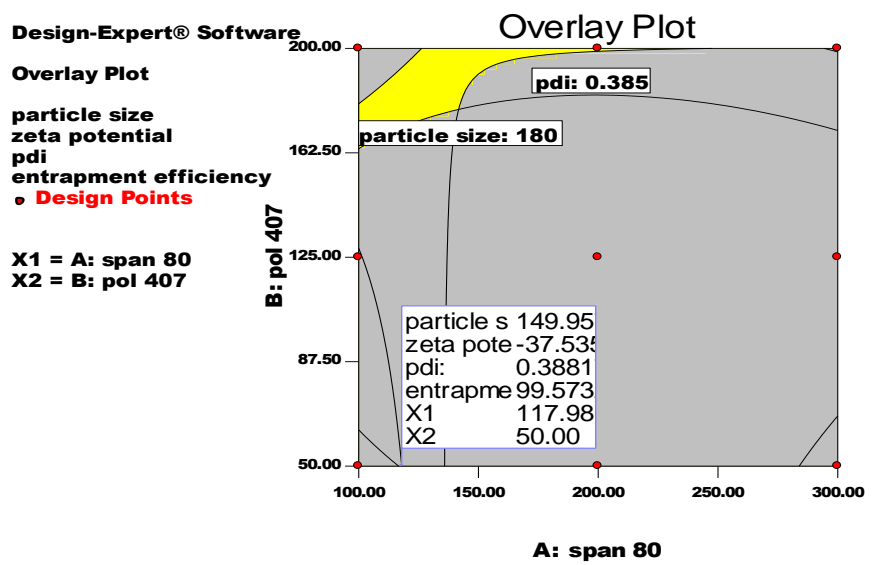

Figure 2. Design space for natamycin cubosomes. Yellow regions depicts the likely permutations to achieve predictable results, Overlay plot of concentration of span 80 and concentration of poloxamer 407.

\subsection{In vitro drug release}

The cumulative percentage drug release of Natamycin from cubosome dispersion $(1 \mathrm{mg} / \mathrm{ml})$ for the optimized batch F7 was compared against marketed eye drop formulation Natadrops ${ }^{\circledR}$ and is as shown in Figure 3.

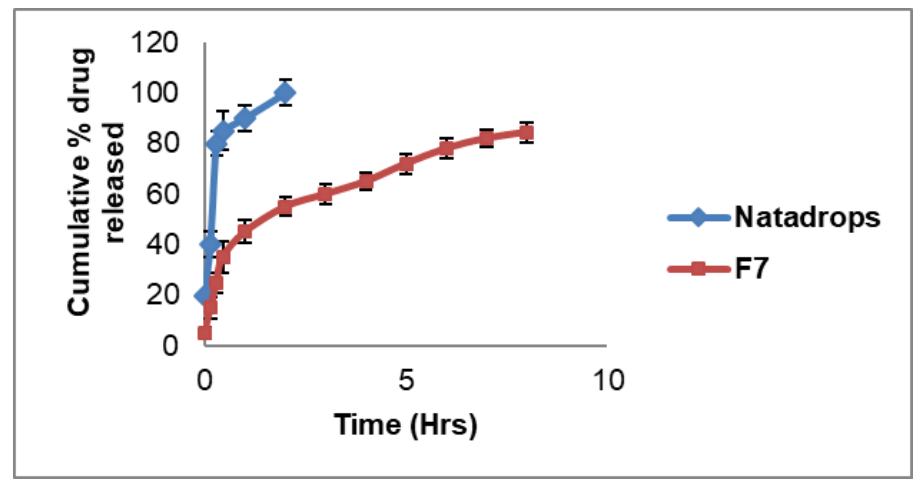

Figure 3. In vitro drug release profile of F7 and Natadrops®.

F7 exhibited a drug release of $84.29 \%$ at the end of 8 hours whereas for the marketed formulation $100 \%$ release was achieved within a time span of 2 hours. The above outcomes correlate with the findings reported elsewhere [1]. Furthermore, the release studies were subjected to kinetic modelling to find the bestfit release model. The release pattern from cubosome nanoparticle was found to be a bifunctionality process with an initial burst release followed by slower sustained release up to $8 \mathrm{~h}$. The burst release is due to the drug adsorbed onto the outer layer of the cubosome nanoparticles and this is necessary for building sufficient levels of drug in the cornea to kill the microbes immediately after administration into the eye $[16,17]$. Prolonged-release of natamycin from cubosomes was ascribed to the sustained release property of lipidic surfactants and is also contributed by its mucoadhesion ability [16].

\subsection{In vitro antifungal activity}

The in vitro antifungal activity of natamycin cubosome was compared with pure drug solution (DMSO) and Natadrops ${ }^{\circledR}$ against Aspergillus fumigatus and Candida albicans (Figure 4).

The results identify the pure drug solution to have the maximum zone of inhibition against both strains of fungi but on statistical evaluation, (ANOVA followed by post hoc Tukey test, Prism 5) no significant difference ( $\mathrm{p}>0.05$ ) was seen in comparison to natamycin cubosomes. Correspondingly, a higher zone of inhibition was obtained for natamycin cubosomes in contrast to Natadrops ${ }^{\circledR}$, thus a significant difference $(p<0.05)$ was seen on statistical examination. Accordingly, the outcomes highlight natamycin cubosomes to have a similar spectrum of antifungal efficacy as seen for pure drug solution. The in vitro 
antifungal activity results are in concurrence with the in vitro antifungal release data; hence, natamycin cubosomes present better prospects for treating ocular fungal keratitis.

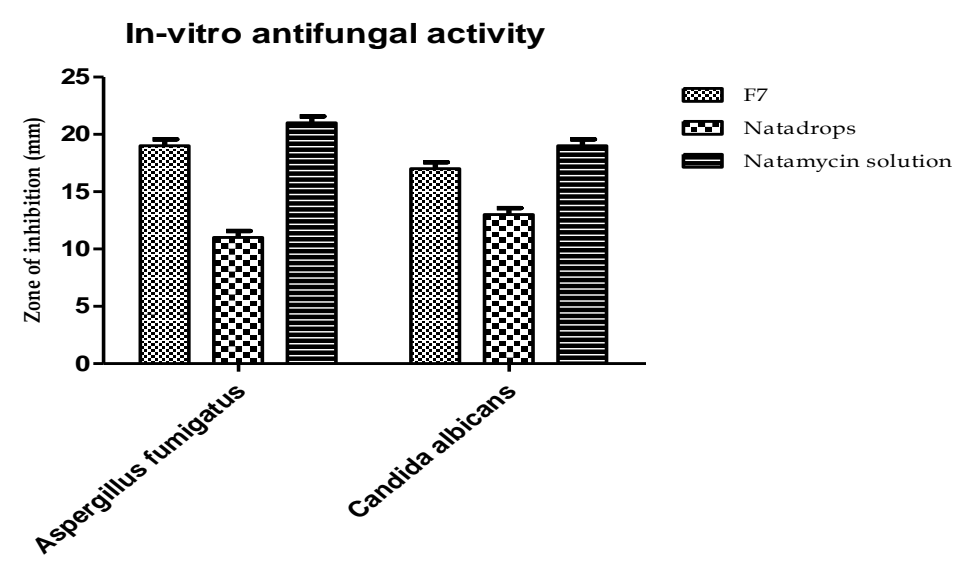

Figure 4. In vitro antifungal activity of F7, Natamycin solution and Natadrops $\AA_{\text {. }}$

\subsection{Polarised light microscopy (PLM)}

PLM images of the optimized natamycin cubosomes are presented in Figure 5. The cross-polarized images are used to study gross morphology of the particles formed and to rule out whether a cubic or hexagonal symmetrical structure is formed. As reported in literature mesophasic cubic structures are isotropic and produce no birefringence thus black background particle images are seen when observed under a cross polarizer, our findings were similar to the reported results [16]. Thus, PLM images confirm the formation of cubosomes.

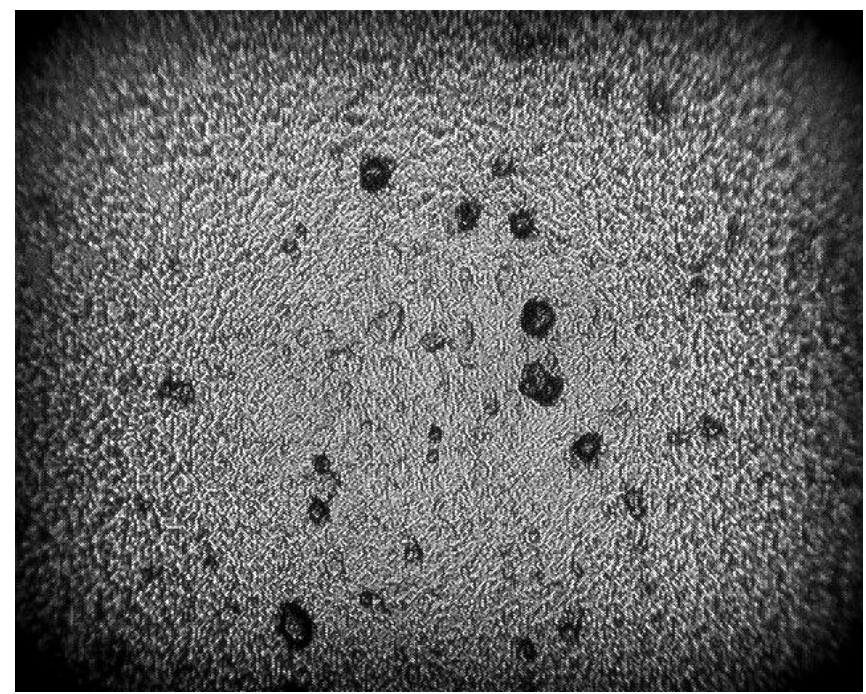

Figure 5. PLM image of optimized natamycin cubosome nanoparticles at $27^{\circ} \mathrm{C}$.

\subsection{Small angle X-ray scattering (SAXS)}

SAXS studies were undertaken to identify the type of mesophasic cubic structure formed, which could not be confirmed by PLM images. Figure 6 shows the scattering profile of optimized natamycin cubosomes.

The scattering profile was investigated for the number of peaks, their width and their relative inter distance, to infer the internal structure of the nanoparticle. The peak ratios $(\sqrt{2}: \sqrt{ } 4: \sqrt{6}: \sqrt{ } 8)$ corresponded to Im3m mesophasic cubic structures [18] and the lattice parameter was found to be $76.48 \mathrm{~nm}$. Furthermore, the particle size was determined to be $155.65 \mathrm{~nm}$ and thus, in concurrence to the particle size noted using zeta sizer $(158.7 \mathrm{~nm})$. 


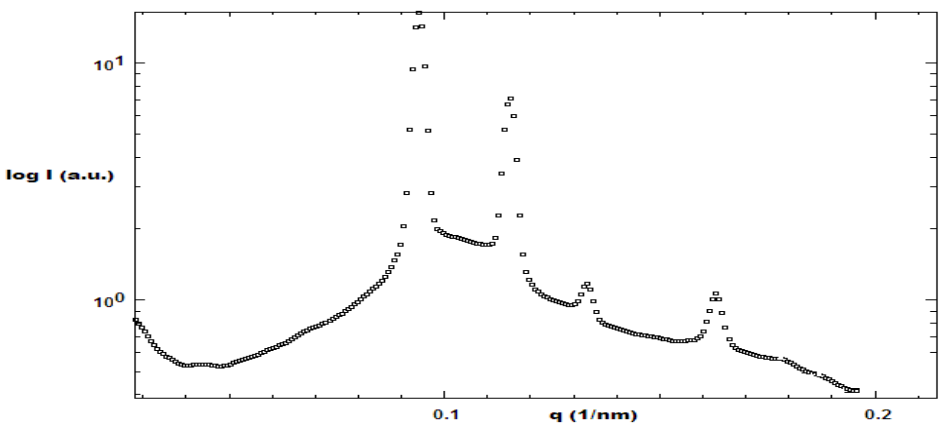

Figure 6. SAXS profile of optimized natamycin cubosome nanoparticles at $27^{\circ} \mathrm{C}$.

\subsection{Field emission gun transmission electron microscopy (FEG TEM)}

TEM image is presented in Figure 7. The images point out the optimized cubical nanoparticles to be irregular cubical symmetry with embedded characteristic water channels, the particles were unaggregated [18]. The particle diameters were in the range $150-200 \mathrm{~nm}$, thus similar to particle size measurement determined by a zeta sizer.

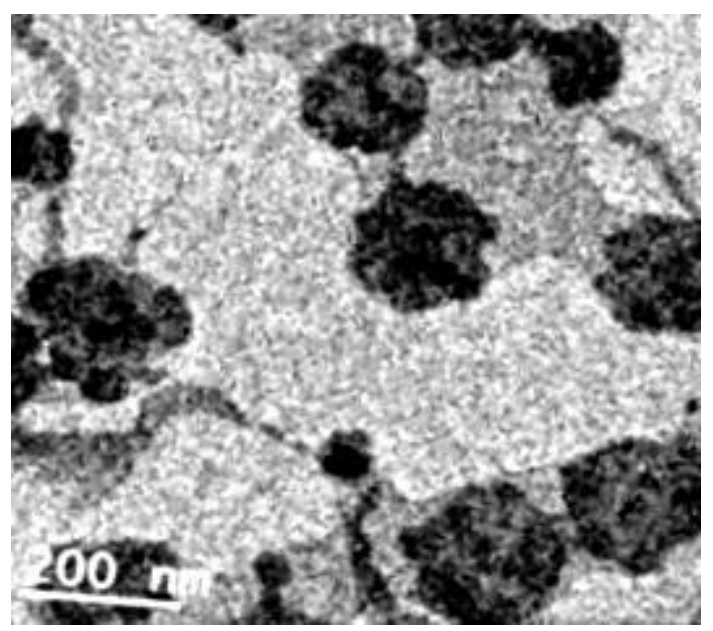

Figure 7. FEG TEM image of optimized formulation.

\section{7. $X$ ray diffraction $(X R D)$}

XRD studies were performed to conclude on the state of the entrapped drug in the cubosome structure. Figure 8 depicts the diffraction spectrum of the pure drug and the optimized natamycin cubosomes.

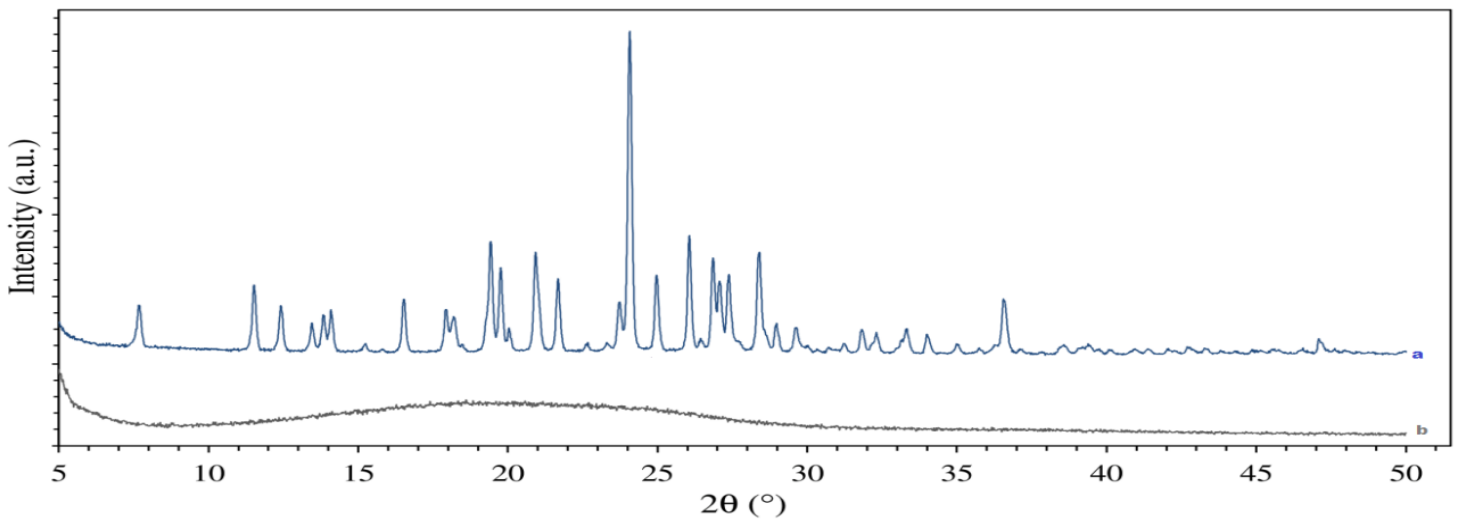

Figure 8. XRD profile of (a) Natamycin (b) optimized formulation. 
The spectra for the pure drug was similar to the reported literature findings and that several different sharp diffraction peaks in the natamycin diffractogram indicated its crystalline form $[19,20]$. In contrast to the pure drug, the optimized cubosome formulation indicated a large decline in diffraction peaks belonging to natamycin. Hence, can be authenticated that the drug was completely entrapped in the cubical structure in amorphous form.

\subsection{Ocular irritancy test}

In vivo eye irritation testing was carried out using rabbit and as per the Draize test. Optimized formulations F7 were used for this test. The formulations were scored to be zero in all observations indicating to be nonirritating (Table 4) with no ocular damage or abnormal clinical signs to the cornea, iris or conjunctivae. Thus, the formulation were found to be fit for the eye instillation.

Table 4. Scoring of optimized formulation for ocular iritancy test as per Draize test.

\begin{tabular}{lcccccc}
\hline Parameters & \multicolumn{6}{c}{ Optimized formulation (F7) } \\
\hline Time (hr) & 1 & 2 & 4 & 24 & 48 & 72 \\
Redness & 0 & 0 & 0 & 0 & 0 & 0 \\
Inflammation & 0 & 0 & 0 & 0 & 0 & 0 \\
Excessive tearing & 0 & 0 & 0 & 0 & 0 & 0 \\
\hline
\end{tabular}

\subsection{Ex vivo corneal permeation studies}

The ex vivo corneal permeation of natamycin from the optimized formulation F7 and drug suspension was studied to determine apparent permeability (Papp) and steady-state flux (Jss). Figure 9 represents the cumulative amount of drug permeated per unit area vs. time. The linear end part of the curve was used to determine the Jss.

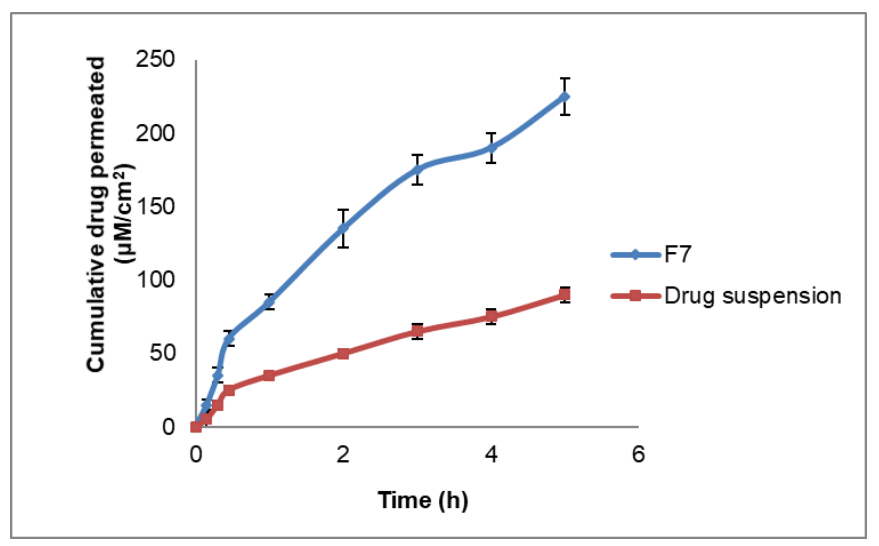

Figure 9. Cumulative drug permeation profile of F7 and drug suspension.

The Papp and Jss for F7 were found to be $20.59 \times 10^{-2} \mathrm{~cm} / \mathrm{hr}$ and $5.46 \mathrm{~mol} / \mathrm{hr}$ respectively. İ contrast the pure drug suspension showed lower values of Papp $\left(6.64 \times 10^{-2} \mathrm{~cm} / \mathrm{hr}\right)$ and Jss $(1.58 \mathrm{~mol} / \mathrm{hr})$. The result reinforces the ability of drug-loaded cubosome nanoparticles in improving corneal absorption of the drug as it could cross the corneal epithelium owing to its lipophilic structuring and nanosize, thus showcased an ability to effectively treat ocular fungal keratitis.

\subsection{Stability studies}

Lyophilized cubosome nanoparticles F7 were evaluated for stability studies. Table 5 presents the evaluation paremeters studied from intial time point to final time point ( 3 months). At the end of final time point the product did not show any signs of melting of the cake, thus was stable. Insignificant changes were seen in the evaluation parameters. The in vitro drug profile was also determined at the end of final time point (Figure $10 \mathrm{c}$ ). The \%release at end of $8 \mathrm{hrs}$ was almost similar $(82 \%)$ to that obtained at the intial stage, thus exhibited insignificant differences $(p>0.05)$. The PLM and TEM images also confirm to no change in 
morphology of the cubosomes at the end of the study (Figure 10a, 10b respectively). Thus, the stability data confirms thermodynamic stability of optimized cubosome nanoparticles.

Table 5. Stability data of optimzed formulation.

\begin{tabular}{lcc}
\hline Parameters & Intial & Final \\
\hline Particle size $(\mathbf{n m})$ & $158.7 \pm 2$ & $162.12 \pm 1.32$ \\
Zeta potential $(\mathrm{mV})$ & $-40 \pm 2.12$ & $-38.82 \pm 1.15$ \\
PDI & $0.328 \pm 0.001$ & $0.332 \pm 0.001$ \\
Entrapment efficiency & $99.85 \pm 0.85$ & $98.12 \pm 0.72$ \\
Description & White powder & White powder \\
\hline
\end{tabular}

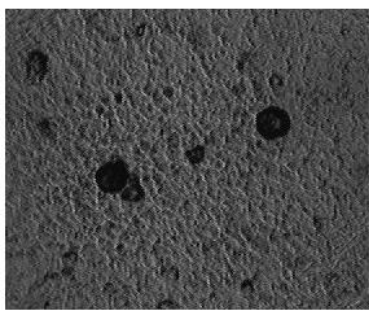

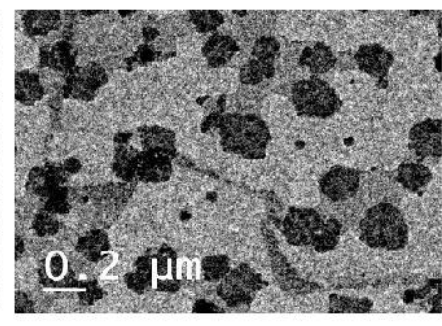

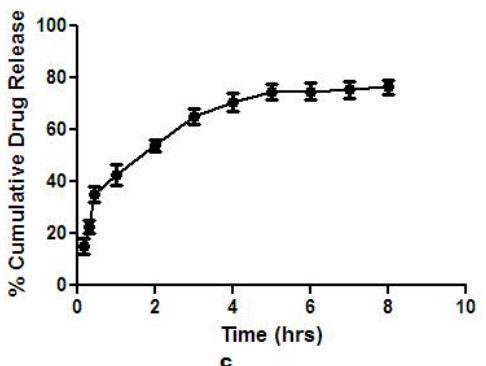

Figure 10. a) PLM image b) FEG TEM image c) in vitro drug release profile for formulation F7 at the end of stability study.

\section{CONCLUSION}

In the present study a $3^{2}$ statistical factorial design was used to prepare optimized natamycin cubosome nanoparticles. Briefly, dry lipidic surfactant films were dispersed into colloidal solution by the aid of ultrasound probe sonication technique. The critical quality attributes like particle size, zeta potential, PDI and entrapment efficiency were significantly affected by the concentration of lipophilic surfactant span 80 and concentration of stabilizer poloxamer 407. The Im $3 \mathrm{~m}$ mesophasic cubic structure formed was affirmed by SAXS profiles and TEM images. XRD studies also confirmed drug entrapped in cubical particles in amorphous form. The optimized natamycin cubosome nanoparticles exhibited enhanced in vitro antifungal activity and precorneal permeation. Moreover, the drug release pattern was biphasic and sustained up to 8 hours. No ocular damage in cornea, conjunctiva or iris was observed. Thus, these cubosome nanocarriers present a propitious delivery system for effective management of ocular fungal keratitis.

\section{MATERIALS AND METHODS}

Natamycin and Cithrol Glycerol monooleate (GMO) HP-SO-LK, were obtained as a gift sample from Bimal Pharma Pvt. Ltd., (Mumbai) and Croda International (Mumbai) respectively. Poloxamer 407 was procured from BASF (Mumbai, India). Dimethyl sulfoxide and span 80 was purchased from Fisher scientific (Mumbai). All other chemicals and reagents used were of AR grade.

\subsection{Formulation and optimization of cubosomes using $3^{2}$ factorial design}

A $3^{2}$ factorial statistical design was used for formulation optimization of cubosomes with three levels, two factors and nine runs were planned using Design Expert ${ }^{\circledR}$ software (Version 11) (Table 1 and Table 6).

Briefly, the concentration of GMO was set to $600 \mathrm{mg}$ for each sample, various concentration of span 80 and poloxamer 407 as denoted in Table 2 was added in to round bottom flask. Thereafter, for complete solubilization of the lipids and stabilizer, chloroform $(5 \mathrm{ml})$ was added so as to obtain one phase system. Later, the second phase containing drug was composed by solubilizing $50 \mathrm{mg}$ of natamycin in DMSO solvent $(1 \mathrm{ml})$, the resulting solution was subsequently add-mixed to the lipidic solution. Chloroform was evaporated in a rotary evaporator held down at a reduced pressure, rotating at $60 \mathrm{rpm}$ and temperature of $60 \pm 2^{\circ} \mathrm{C}$, leading to the accumulation of a thin film at the bottom of the flask [21]. PBS buffer saline ( $\mathrm{pH}=7.4$, 
$50 \mathrm{ml}$ ) was added to the dry film and further subjected to sonication using a probe sonicator (Sonics Vibra cell VCX 500) for $10 \mathrm{~min}$ at $25^{\circ} \mathrm{C}, 70 \%$ amplitude with a cycle of 3 pulse interrupted by 7 sec breaks [21,22]. Natamycin dispersion was microcentrifuged (Remi motors, RM 12-C) at $12000 \mathrm{rpm}$ (30 mins) with repetitive washings to eliminate the unentrapped drug, and were stored in a vial at R.T $\left(25^{\circ} \mathrm{C}\right)$ until further evaluation.

Table 6. $3^{2}$ Factorial design.

\begin{tabular}{llll}
\hline \multirow{2}{*}{ Factor } & \multicolumn{2}{l}{ Levels } & \\
\cline { 2 - 4 } & $\mathbf{- 1}$ & $\mathbf{0}$ & $\mathbf{1}$ \\
\hline $\mathbf{X}_{\mathbf{1}}$-Concentration of Span $\mathbf{8 0}(\mathbf{m g})$ & 100 & 200 & 300 \\
$\mathbf{X}_{\mathbf{2}}$-Concentration of P-407 (mg) & 50 & 125 & 200 \\
\hline
\end{tabular}

\subsection{Characterization}

\subsubsection{Particle size, polydispersibility index (PDI) and zeta potential}

The particle size of formulated batches was determined using Malvern Zetasizer Nano Series (Malvern Instruments, Malvern, India). $1 \mathrm{ml}$ of cubosomal suspension was diluted by $10 \mathrm{ml}$ of double distilled water to estimate mean particle size, PDI and zeta potential.

\subsubsection{Drug entrapment efficiency}

The prepared dispersion was positioned into a centrifuge device and was centrifuged at 12,000 rpm for $30 \mathrm{~min}$. The supernatant was analyzed by UV spectrophotometer (UV 1800, Shimadzu Japan) to determine the free drug concentration $\left(C_{\text {free }}\right)$ at $304 \mathrm{~nm}$ and dilution was done if required [23]. The \%drug entrapped (EE) was calculated using the following equation:

$$
\% \mathrm{EE}=\frac{\mathrm{C} \text { (total drug concentration })-\mathrm{C}(\text { free drug })}{\mathrm{C}(\text { total drug concentration })}
$$

\subsubsection{In vitro drug release}

In vitro drug release study of natamycin cubosome dispersion was carried out by using Franz diffusion cell. The formulation was placed in the donor compartment \& freshly prepared phosphate buffer (PBS) pH 7.4 was placed in a receptor compartment. Cellophane membrane, previously soaked overnight in PBS pH 7.4, was placed in between the donor and receptor compartment [24]. The assembly was placed on a thermostatically controlled magnetic stirrer and the temperature of the medium was maintained $\left(37^{\circ} \mathrm{C} \pm\right.$ $\left.0.5^{\circ} \mathrm{C}\right) .1 \mathrm{ml}$ sample was withdrawn at a predetermined time interval and the sample volume of fresh medium was replaced. The withdrawn sample was diluted with PBS pH 7.4 and analyzed by UV spectrophotometer (Shimadzu UV1800, Japan) at $304 \mathrm{~nm}$. The release data were fitted in various kinetic models like zero order, first order, Higuchi, Korsmeyer-Peppas model to identify the best fit model.

\subsubsection{In vitro antifungal activity}

The in vitro antifungal activity of the optimized cubosomal dispersion was determined against Aspergillus fumigatus (NCIM 902) and Candida albicans (ATCC 18804). Culture suspension of Candida albicans was made from fresh cultures (2 day old) grown on Sabouraud dextrose agar (SDA) slants, by suspending one loop full of culture in sterile water $(5 \mathrm{ml})$ and vortexed $(15 \mathrm{~s})$ to obtain a homogenous suspension. The optical density of the resulting culture was set at a value 1, measured by Elico Spectro colorimeter CL 153 at $600 \mathrm{~nm}$. Aspergillus fumigatus culture suspension was composed from fresh, fully grown (3-5 day old) cultures on SDA slants [25]. The colonies were flooded with distilled sterile water (5 ml). Tween $20(1 \%)$ was added to assist the dispersion of Aspergillus. The colonies were scraped with a sterile loop; the isolates were then shaken briskly for 15 secs by means of a vortex mixer and then moved to a sterile tube. The optical density of the suspensions was set as 0.13 , measured by UV spectrophotometer (Shimadzu UV-1600) at 530 $\mathrm{nm}$ [25]. Agar well diffusion method was used to examine anti-fungal activity, $1 \mathrm{ml}$ of microbial inoculum was seeded into SDA medium and poured into Petri plates, a sterile cork borer of diameter $6 \mathrm{~mm}$ were punched aseptically to create a well and cubosome dispersion sample $(0.1 \mathrm{ml})$ was added into the well. The plates were incubated for $24 \mathrm{hrs}, 27^{\circ} \mathrm{C}$ (Candida albicans) and $48 \mathrm{hrs}, 27^{\circ} \mathrm{C}$ (Aspergillus fumigatus). The zone of 
inhibition from the optimized cubosome nanoparticles was compared with pure drug solution (DMSO) and pure drug suspension ( $\mathrm{pH} 7.4)$.

\subsubsection{Polarized light microscopy (PLM)}

Optimized natamycin cubosomewere observed under PLM (Carl Zeiss, Jena, Germany), a drop of cubosome dispersion was placed on a glass slide and observed under PLM $\left(27^{\circ} \mathrm{C}\right)$ to identify whether cubosomes or hexosomes were produced, as the nanoparticle morphology.

\subsubsection{Small angle X-ray scattering (SAXS)}

The scattering profiles were measured on Xeuss 2 (Xenocs SAS, France), consisting of an Eiger R $1 \mathrm{M}$ as the detector, GeniX3D Cu 30 Watts $\mathrm{Cu}$ tube with $50 \mathrm{KV} 0.6 \mathrm{~mA}$ current, $1.54 \AA$ as the radiation wavelength and the scattering angle ranged as $0.2^{\circ}$ to $2.8^{\circ}$. The measurements were done in a vacuum $\left(27 \pm 0.1^{\circ} \mathrm{C}\right)$. The scattering patterns were transformed to plots of intensity vs. q-value, to identify the peak point, and its Miller Indices to distinguish the phase structure using SAXS processing software [Scatter developed by S. Förster (University of Hamburg), and L. Apostol(DUBBLE/ESRF)].

\subsubsection{Field emission gun transmission electron microscopy (FEG TEM)}

The samples were negatively stained in $1 \%(\mathrm{w} / \mathrm{v})$ phosphotungstic acid (3-5 min) [16]. A droplet (5- $\mu \mathrm{l})$ of the optimized cubosome suspension was positioned onto a 300 mesh carbon coated copper grid and was allowed to settle (3-5 min). The excess fluid was wipped off by the aid of absorbent paper. Later, envisaged on a FEI Model Tecnai G2, F30 300KV (FEI-USA) and the image were captured on a Gatan axis-mount 2k×2k digital camera.

\subsubsection{X-Ray diffractometer}

XRD studies were conducted on a PW3040/60 X'pert PRO (PANalytical, Netherlands). Cu X-ray tube operating at $45 \mathrm{kV}$ and $40 \mathrm{~mA}$ produced incident X-radiation. Diffraction images were obtained on an X'Pert data collector. Samples such as natamycin and optimized natamycin cubosome nanoparticles were analysed at $25^{\circ} \mathrm{C}$ over the $2 \mathrm{q}$ range $2-50^{\circ}$ with a step size of $0.05^{\circ}(60$ secs).

\subsubsection{Ocular irritancy test}

Ocular irritancy test was performed on optimized natamycin cubosomes according to Draize test $[1,18]$. The formulation ( 1 to 2 drops) was placed into the right eye of the rabbit. The left eye served as control and was treated with saline. The ocular conditions were recorded at 1, 2, 4, 24, 48, and 72 hours after the last administration the detection of endpoint was based on acute toxicity symptoms viz. redness, inflammation and tear flux over a period of 72 hours. In accordance with the Draize method, ocular irritation scores for every rabbit are calculated by adding the irritation scores for the cornea, iris and conjunctiva. The eye irritation score is calculated by dividing the total score for all rabbits by the number of rabbits $[1,18]$. Irritation was classified according to four grades: practically non-irritating, score $0-3$; slightly irritating, score 4-8; moderately irritating, score 9-12; and severely irritating (or corrosive), score 13-16 (IAEC approval for the tests was obtained Ref No; CPCSEA/IAEC/P'ceutics-33/2017-18/132).

\subsubsection{Ex vivo corneal permeation studies}

The corneal permeation efficacy of the optimized natamycin cubosomes was performed on freshly excised goat corneas obtained from local slaughter house [26]. Whole goat eyeballs were placed in cold artificial tear saline $\left(4^{\circ} \mathrm{C}\right)$. The cornea along with small layer $(5-6 \mathrm{~mm})$ of a butting scleral tissue was carefully excised, washed several times with cold saline, and thereafter stored in cold artificial tear buffer saline (pH 7.4) until further use. Drug permeation studies were undertaken on Franz diffusion cell for optimized cubosome formulation and natamycin saline suspension. The samples were placed in the donor compartment in close proximity with the epithelial side of the cornea, the top of the cell was completely closed and the acceptor compartment was filled with artificial tear fluid. The drug permeation study was planned for 6 hours with continuous magnetic stirring $\left(100 \mathrm{rpm}, 37^{\circ} \mathrm{C} \pm 0.5^{\circ} \mathrm{C}\right)$. Sampling was done at prespecified time intervals, aliquots were removed from the acceptor compartment and replaced with the same volume of artificial tear fluid, so as to maintain sink conditions. The samples after further dilutions were subsequently analyzed by UV spectrophotometer (UV 1800, Shimadzu Japan) at $304 \mathrm{~nm}$ to measure drug content. Apparent permeability coefficient (Papp) and steady-state flux (Jss) of the drug was calculated using the following equation: 


$$
\operatorname{Papp}=\frac{\Delta Q}{\Delta t} x \frac{1}{A C_{0}}
$$

Where, $\Delta \mathrm{Q} / \Delta \mathrm{t}$ is the steady-state flux $(\mu \mathrm{mol} / \mathrm{h}), \mathrm{C}_{0}$ is the initial concentration in the donor chamber $(\mu \mathrm{mol} / \mathrm{mL})$, and $\mathrm{A}$ is the surface area of the corneal layer $\left(\mathrm{cm}^{2}\right)$.

\subsubsection{Stability studies}

The optimized formulation F7 was subjected to stability studies. Lyophilized formulation were added in vials, secured with rubber stoppers equipped with flip off seal and was charged in stability chamber (Thermolabs scientific equipments, India) for 3 months at temperature of $25 \pm 2^{\circ} \mathrm{C}$ and $\mathrm{RH} 60 \pm 5 \%$. The stability tests parameters inspected were physical description, particle size, zeta potential, PDI and entrapment efficiency. Additionally, the samples were also analyzed for in vitro drug release profiling, PLM and FEG TEM at the end of final time point.

Acknowledgements: The authors are thankful to Bimal Pharma Pvt. Ltd., (Mumbai, India) for providing Natamycin and Croda, (Mumbai, India) for generous supply of Cithrol GMO-HP-SO-LK.The authors thank SAIF, IIT-Bombay (Maharashtra, India) and Wockhardt Ltd. (Aurangabad, India) for rendering provisions to use SAXS facility and XRD respectively.

Author contributions: Concept - K.M., D.MH.; Design - K.M., D.R.; Supervision - D.MH.; Resources -D.MH., K.M.; Materials - K.M.; Data Collection and/or Processing - K.M., D.R.; Analysis and/or Interpretation - D.MH., K.M., D.R.; Literature Search - K.M., D.R.; Writing - K.M., D.R.; Critical Reviews - D.MH., K.M.,D.R.

Conflict of interest statement: The authors declared no conflict of interest in the manuscript.

\section{REFERENCES}

[1] Chandasana H, Prasad YD, Chhonker YS, Chaitanya TK, Mishra NN, Mitra K, Shukla PK, Bhatta RS. Corneal targeted nanoparticles for sustained natamycin delivery and their PK/PD indices: an approach to reduce dose and dosing frequency. Int J Pharm. 2014; 477(1-2): 317-325. [CrossRef]

[2] Ansari Z, Miller D, Galor A. Current Thoughts in Fungal Keratitis: Diagnosis and Treatment. Curr Fungal Infect Rep. 2013; 7(3): 209-218. [CrossRef]

[3] Kaur IP, Kanwar M. Ocular preparations: the formulation approach. Drug Dev Ind Pharm. 2002; 28(5): 473-493. [CrossRef]

[4] Hamalainen KM, Kananen K, Auriola S, Kontturi K, Urtti A. Characterization of paracellular and aqueous penetration routes in cornea, conjunctiva, and sclera. Invest Ophthalmol Vis Sci. 1997; 38(3): 627-34.

[5] Keister JC, Cooper ER, Missel PJ, Lang JC, Hager DF. Limits on optimizing ocular drug delivery. J Pharm Sci. 1991; 80(1): 50-53. [CrossRef]

[6] Shen HH, Chan EC, Lee JH, Bee YS, Lin TW, Dusting GJ, et al. Nanocarriers for treatment of ocular neovascularization in the back of the eye: new vehicles for ophthalmic drug delivery. Nanomed. 2015; 10(13): 20932107. [CrossRef]

[7] Boyd BJ, Dong Y-D, Rades T. Nonlamellar liquid crystalline nanostructured particles: advances in materials and structure determination. J Liposome Res. 2009; 19(1): 12-28. [CrossRef]

[8] Chung H, Kim J, Um JY, Kwon IC, Jeong SY. Self-assembled "nanocubicle" as a carrier for peroral insulin delivery. Diabetologia. 2002; 45(3): 448-451.[CrossRef]

[9] Lalu L, Tambe V, Pradhan D, Nayak K, Bagchi S, Maheshwari R, Kalia K,Tekade RK. Novel nanosystems for the treatment of ocular inflammation: Current paradigms and future research directions. J Control Release Off J Control Release Soc. 2017; 268: 19-39. [CrossRef]

[10] Almeida H, Amaral MH, Lobao P, Frigerio C, Sousa Lobo JM. Nanoparticles in Ocular Drug Delivery Systems for Topical Administration: Promises and Challenges. Curr Pharm Des. 2015; 21(36): 5212-5224. [CrossRef].

[11] Dong Y, Chang Y, Qian W, Tong J, Zhou J. Effects of surfactants on size and structure of amylose nanoparticles prepared by precipitation. Bull Mater Sci. 2016; 39(1): 35-39. [CrossRef] 
[12] Hu FQ, Jiang SP, Du YZ, Yuan H, Ye YQ, Zeng S. Preparation and characterization of stearic acid nanostructured lipid carriers by solvent diffusion method in an aqueous system. Colloids Surf B Biointerfaces. 2005; 45(3-4): 167173. [CrossRef]

[13] Zirak M, Pezeshki A. Effect of Surfactant Concentration on the Particle Size, Stability and Potential Zeta of Beta carotene Nano Lipid Carrier. IntJCurrMicrobiolAppSci. 2015; 4: 924-932.

[14] McClements DJ. Crystals and crystallization in oil-in-water emulsions: implications for emulsion-based delivery systems. Adv Colloid Interface Sci. 2012; 174: 1-30. [CrossRef]

[15] Hao J, Fang X, Zhou Y, Wang J, Guo F, Li F, Peng X. Development and optimization of solid lipid nanoparticle formulation for ophthalmic delivery of chloramphenicol using a Box-Behnken design. Int J Nanomedicine. 2011; 6: 683-692. [CrossRef]

[16] Ali Z, Sharma P, Warsi M. Fabrication and Evaluation of Ketorolac Loaded Cubosome for Ocular Drug Delivery. J Appl Pharm Sci. 2016; 6: 204-208. [CrossRef]

[17] Magenheim B, Levy MY, Benita S. A new in vitro technique for the evaluation of drug release profile from colloidal carriers - ultrafiltration technique at low pressure. Int J Pharm. 1993; 94(1): 115-123. [CrossRef]

[18] Han S, Shen J, Gan Y, Geng H, Zhang X, Zhu C, et al. Novel vehicle based on cubosomes for ophthalmic delivery of flurbiprofen with low irritancy and high bioavailability. Acta Pharmacol Sin. 2010; 31(8): 990-998. [CrossRef]

[19] Cevher E, Sensoy D, Zloh M, Mulazimoglu L. Preparation and characterisation of natamycin: gamma-cyclodextrin inclusion complex and its evaluation in vaginal mucoadhesive formulations. J Pharm Sci. 2008; 97(10): 4319-4335. [CrossRef]

[20] Liu Q, Wu X, Qian F, Zhang T, Mu G. Influence of natamycin loading on the performance of transglutaminase- induced crosslinked gelatin composite films. Int J Food Sci Technol.2019; 54(7): $2425-2436$. [CrossRef]

[21] Bei D, Marszalek J, Youan B BC. Formulation of dacarbazine-loaded cubosomes-part I: influence of formulation variables. AAPS PharmSciTech. 2009; 10(3): 1032-1039. [CrossRef]

[22] Ali MA, Noguchi S, Iwao Y, Oka T, Itai S. Preparation and Characterization of SN-38-Encapsulated Phytantriol Cubosomes Containing alpha-Monoglyceride Additives. Chem Pharm Bull (Tokyo). 2016; 64(6): 577-584. [CrossRef]

[23] El Nabarawi MA, Abd El Rehem RT, Teaima M, Abary M, El-Mofty HM, Khafagy MM, Lofty NM,Salah M. Natamycin niosomes as a promising ocular nanosized delivery system with ketorolac tromethamine for dual effects for treatment of candida rabbit keratitis; in vitro/in vivo and histopathological studies. Drug Dev Ind Pharm. 2019; 45(6): 922-936. [CrossRef]

[24] Abdelbary G, El Gendy N. Niosome-encapsulated gentamicin for ophthalmic controlled delivery. AAPS PharmSciTech. 2008; 9(3): 740-747. [CrossRef]

[25] Petrikkou E, Rodriguez T JL, Cuenca EM, Gomez A, Molleja A, Mellado E. Inoculum standardization for antifungal susceptibility testing of filamentous fungi pathogenic for humans. J Clin Microbiol. 2001; 39(4): $1345-1347$. [CrossRef]

[26] Khames A, Khaleel MA, El-Badawy MF, El-Nezhawy AOH. Natamycin solid lipid nanoparticles - sustained ocular delivery system of higher corneal penetration against deep fungal keratitis: preparation and optimization. Int J Nanomedicine. 2019; 14: 2515-2531. [CrossRef] 